\title{
Optimized strategy for the calibration of superconducting gravimeters at the one per mille level
}

Article in Journal of Geodesy · September 2015

DOI: $10.1007 / \mathrm{s} 00190-015-0856-7$

CITATIONS

2

4 authors, including:

\section{Michel Van Camp}

Royal Observatory of Belgium

110 PUBLICATIONS 957 CITATIONS

SEE PROFILE

Olivier De Viron

Université de La Rochelle

191 PUBLICATIONS 1,203 CITATIONS

SEE PROFILE
READS

60

Bruno Meurers

University of Vienna

77 PUBLICATIONS 665 CITATIONS

SEE PROFILE

Some of the authors of this publication are also working on these related projects:

Project

Gravity and hydrogeology View project

Project

Intraplate seismology View project 


\section{Optimized strategy for the calibration of superconducting gravimeters at the one per mille level}

Michel Van Camp ${ }^{1}$, Bruno Meurers ${ }^{2}$, Olivier de Viron ${ }^{3}$ and Thomas Forbriger ${ }^{4}$

1. Royal Observatory of Belgium, Avenue Circulaire, 3, BE-1180 Uccle, Belgium Phone: +32 23730265 Fax: +32 23730339 mvc@oma.be

2. University of Vienna, Department of Meteorology and Geophysics, AT-1090 Wien, Althanstrasse 14, UZA II, 2D504

3. Université de La Rochelle-CNRS UMR 7266, Littoral Environnement et Sociétés (LIENSs), bâtiment ILE, 2, rue Olympe de Gouges, FR-17000 La Rochelle, France

4. Observatorium Schiltach (BFO), Heubach 206, D-77709 Wolfach, Germany \& Geophysikalisches Institut, Karlsruher Institut für Technologie (KIT)

Keywords: Calibration; Superconducting Gravimeter; Absolute Gravimeter; Earth Tide Journal of Geodesy (2015), DOI: 10.1007/s00190-015-0856-7 


\section{Abstract}

This paper reports on different sources of errors that occur in the calibration process of a superconducting gravimeter (SG), determined by comparison with a ballistic absolute gravimeter (AG); some of them have never been discussed in the literature. We then provide methods to mitigate the impact of those errors, to achieve a robust calibration estimate at the $1 \%$ level.

We demonstrate that a standard deviation at the level of $1 \%$ o can be reached within 48 hours by measuring at spring tides and by increasing the AG sampling rate. This is much shorter than what is classically reported in previous empirical studies. Measuring more than five days around a tidal extreme does not improve the precision in the calibration factor significantly, as the variation in the error as a function of $1 / \sqrt{N}$ does not apply, considering the decrease in signal amplitude due to the tidal modulation. However, we investigate the precision improvement up to 120 days, which can be useful if an AG is run continuously: at mid-latitude it would require 21 days to ensure a calibration factor at the $1 \%$ level with a $99.7 \%$ confidence interval. We also show that restricting the AG measurement periods to tidal extrema can reduce instrument demand, while this does not affect the precision on the calibration factor significantly. Then, we quantify the effect of high microseismic noise causing aliasing in the AG time series. We eventually discuss the attenuation bias that might be induced by noisy time series of the superconducting gravimeter.

When experiments are performed at the $1 \%$ o level, 7 are needed to ensure that the error in the calibration estimate will be at the 1 per mille level with a $99 \%$ confidence.

\section{Introduction}

The superconducting gravimeter (SG) is presently the most precise instrument to determine the time fluctuation of local gravity. The measure itself is the voltage controlling the current in a feedback coil in order to keep the equilibrium of a superconducting sphere between the gravity and the magnetic levitation. This voltage is converted into gravity changes using amplitude and phase calibration factors. A precise calibration of the superconducting gravimeters in amplitude is required to constrain oceanic tidal loading models or to evaluate the recent global Earth models, which do not differ by more than $1 \%$ in their tidal gravimetric factors and only 0.01 degree -or equivalently $1.2 \mathrm{~s}$ at the frequency of 2 cycles per day- in the phase (Baker and Bos, 2003). Hence, it is advisable to achieve a calibration with a precision level of one per mille in amplitude and 0.01 degree in phase.

In practice, the phase can be estimated at the $0.01 \mathrm{~s}$ level by measuring the instrument response to step or sine waves (Van Camp et al., 2000). Nowadays, the amplitude factor is classically determined using side-by-side measurements from an absolute gravimeter (AG). The tidal signal allows a determination of the calibration factor at the 1\%o level, given its large amplitude, about 20 times larger than the influence of the atmosphere, the hydrosphere or the polar motion. Other methods, moving a known mass around the SG (Achilli et al. 1995), installing the SG on a calibration platform (Richter, 1995), or comparing with spring gravimeters (Meurers 2012), also allows an estimation of the calibration factor at the $1 \%$ level.

Francis (1997) already noticed that a strong tidal signal is required to obtain a good precision and could achieve the $1 \%$ level in lesser than two days when the tidal signal was strong. Hinderer et al. (1998) could achieve the $1.5 \%$ level after 6.5 days. Then, Francis et al. (1998) reported that at least 5 to 7 days of measurements side-by-side with an $A G$ are required to reach the 1\%o precision level on the SG calibration factor, but in that study they could not benefit from time series starting at a 
tidal extremum. Combining data from more calibration experiments allows a calibration below the 1\%o precision (Rosat et al. 2009; Meurers et al. 2012; Virtanen et al. 2014).

Presently, the calibration precision of SGs operating in the framework of the Global Geodynamics Project (GGP) (Crossley and Hinderer 2009) reaches a few per mille (Meurers 2001). Clearly, as discussed by Rosat et al. (2009), Meurers (2012) and Virtanen et al. (2014), different calibration experiments on the same instrument can give results that differ by more than $1 \%$. As far as we know, this is the first study quantifying the number of experiments needed to achieve one per mille level with a given confidence interval, by investigating the causes of the uncertainties on the amplitude of the calibration factor, and propose methods to mitigate their impact. In particular, we quantify the aliasing effect affecting AG measurements and the attenuation bias caused by noisy SG series.

\section{Effect of the noise on the absolute gravity measurements}

The calibration factor $\beta$ is computed by a least-square fit (LSQ) on the observation equations:

$$
y_{i}=\beta x_{i}+P\left(t_{i}\right)+\varepsilon_{i}
$$

where $x_{i}$ and $y_{i}$ represent the SG and AG times series, respectively $\left(i=1, \ldots, N . \varepsilon_{i}\right.$ are the measurement errors on the AG time series, called drop to drop scatter. As this is usually a Gaussian white noise (Van Camp et al. 2005), we call it GW noise here after. $P\left(t_{i}\right)$ is a first or second degree polynomial, which accounts for the differential instrumental drifts between both instruments (Imanishi et al. 2002; Meurers 2012) and which is estimated by the least-squares fit simultaneously with $\beta$. If the drift is not accounted for, the calibration factor is biased as shown by e.g. Hinderer et al. (1991), Francis and Hendrickx (2001) or Meurers (2012).

\subsection{Amplitude of the tidal signal}

To enhance the signal to noise ratio (SNR), calibrations should be performed at spring tides, where the gravity variation amplitude reaches its maximum. As the tide amplitude decreases, this will cancel out the increase of the factor $\sqrt{N}$ at a given stage. As an example, we generated a 120 daylong synthetic tide at the Membach station (Belgium, $50.61^{\circ} \mathrm{N}, 6.01^{\circ} \mathrm{E}$ ), with a starting date three days before spring tides, and added a Gaussian white noise distributed with a zero mean and 70 $\mathrm{nm} / \mathrm{s}^{2} \mathrm{rms}$ amplitude, consistent with a typical GW. The sampling rate is 1 data (or drop) per $10 \mathrm{~s}$. We then fitted this series on the synthetic tide acting as an SG time series, and calculated the error on the calibration factor provided by the LSQ process as a function of the length of the time series. This is shown by the black curve on Figures 1 a and $1 b$, where it is compared with the $1 / \sqrt{N}$ function shown in red. This red curve is normalized, only the comparison of rates of change is meaningful. One can see that the $1.0 \%$ and $0.5 \%$ o levels of precision are reached after 1.5 and 5 days, respectively. Note that this precision depends linearly on the drop to drop standard deviation, as pointed out by Hinderer et al. (1998).

Figures $1 \mathrm{a}$ and $1 \mathrm{~b}$ also evidence the step-like decrease in the error, where the decrease in the error slows down every 14 days, at neap tides. In other words, as discussed by Francis (1997), the standard deviation of the estimator of the calibration factor decreases when the tidal signal is large. Note that on Figure $1 \mathrm{~b}$, the error first decreases faster than $1 / \sqrt{N}$, because the amplitude of the tide increases over the first three days. 
In our example, less than 5, 42 and 98 days are required to achieve respectively the $1 \%, 0.5 \%$ and $0.3 \%$ level of precision, provided that the GW remains lower than $135 \mathrm{~nm} / \mathrm{s}^{2}$, for peak to peak amplitude of the tidal signal of about $2000 \mathrm{~nm} / \mathrm{s}^{2}$ during those 5 days. Using real data, Francis (1997) could achieve the $1 \%$ level in lesser than 2 days when the tidal signal was maximum, while Francis et al. (1998) obtained error bars at the level of 1\%o starting from day \#7. This is consistent with our simulations, considering their experiment parameters: GW equal to $63.5 \mathrm{~nm} / \mathrm{s}^{2}$ and 100 -drop sets observed hourly at a rate of 1 drop by 10 seconds (Figure 2 ). If this calibration experiment had started at day \#8, corresponding to a tidal maximum of $2550 \mathrm{~nm} / \mathrm{s}^{2}$, our simulation shows that 2.8 days would have sufficed for a same GW of $63.5 \mathrm{~nm} / \mathrm{s}^{2}$.

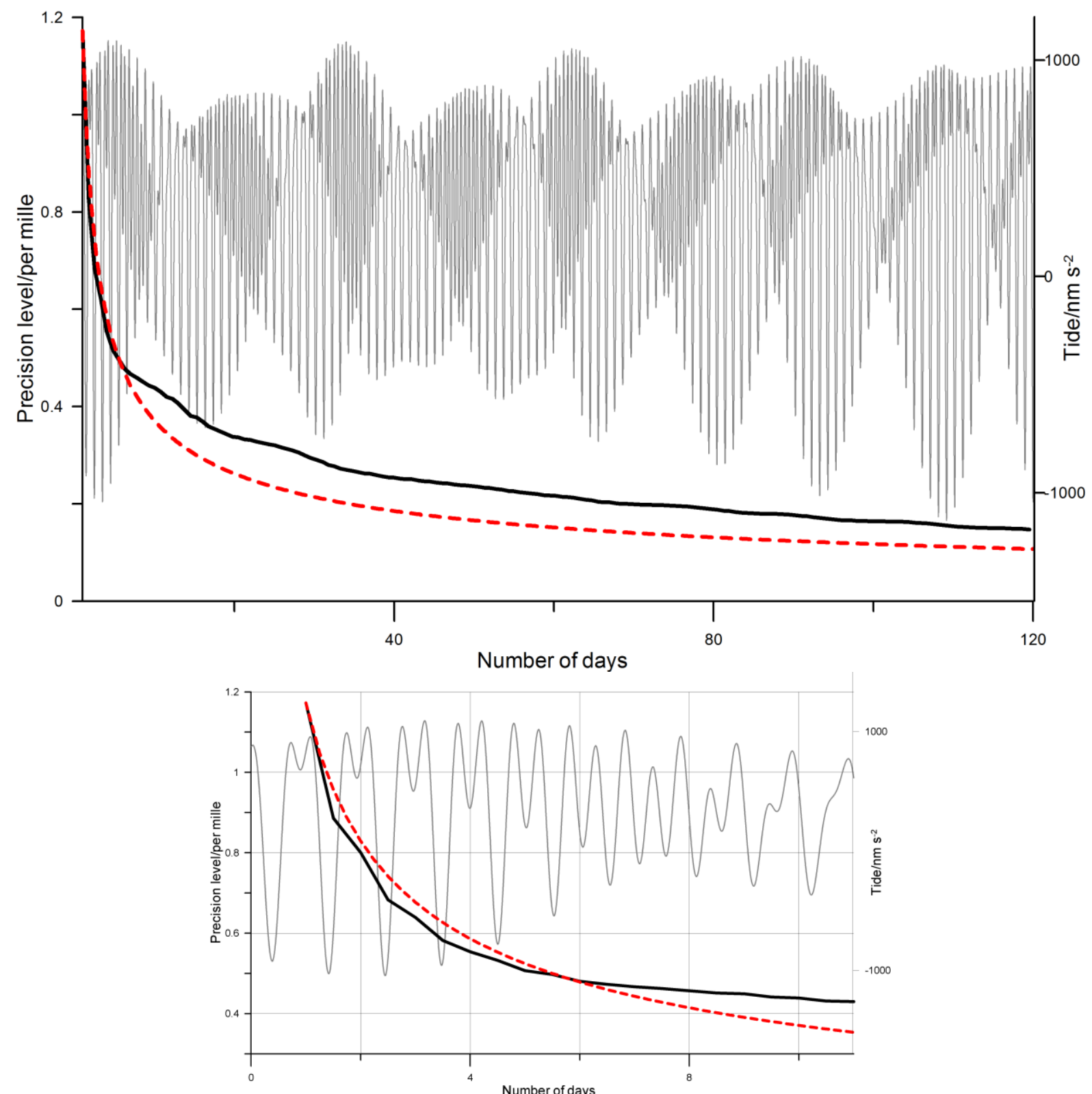

Fig. 1(a) In grey, the tidal signal simulated at the Membach station (Belgium, $50.61^{\circ} \mathrm{N}, 6.01^{\circ} \mathrm{E}$, days since 2014-07-08 00:00). AG series synthesized by adding a GW noise of amplitude $70 \mathrm{~nm} / \mathrm{s}^{2}$ to the tidal signal (10 s sampling interval, continuously). In black, evolution of the error on the calibration [\%] as a function of the number of days. In red, the $1 / \sqrt{N}$ law normalized on the first value of the standard deviation. Due to this normalization, only the comparison of rates of change is meaningful Fig. 1(b) Same as (a) focusing on the 11 first days 


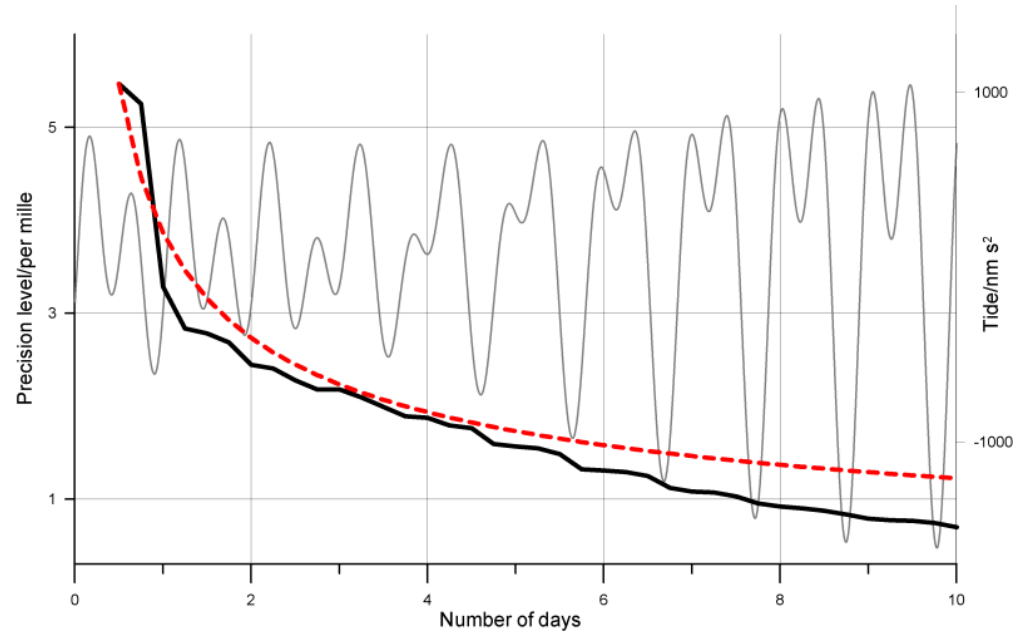

Fig. 2 In grey the tidal signal simulated at the Boulder station (USA, $40.13^{\circ} \mathrm{N},-105.20^{\circ} \mathrm{E}$, days since 1996-07-20 00:00). AG series synthetized by adding a GW noise of amplitude $63.2 \mathrm{~nm} / \mathrm{s}^{2}$ to the tidal signal (10 s sampling interval, 100 drops per set, one set per hour as in Francis et al. (1998)). In black evolution of the error on the calibration [\%] as a function of the number of days. In red, the $1 / \sqrt{N}$ law normalized on the first value of the standard deviation. Due to this normalization, only the comparison of rates of changes is meaningful

\subsection{Measuring during tidal extremes}

As the noise level of the SG does not depend on the gravity value, the signal to noise ratio is maximum when the gravimetric tide reaches its extrema. In the same experiment as in the example here above, we used the full time series and only kept gravity amplitudes around gravity extremes, as shown on Figure 3. This was done considering one AG measurement per $5 \mathrm{~s}$ and one per $10 \mathrm{~s}$, for an AG GW noise with a standard deviation of $62 \mathrm{~nm} / \mathrm{s}^{2}$. The errors, in per mille, are given in Table 1, for 10000 runs.

For a given number of drops, we can reach the same precision of $0.46 \%$ by doubling the sampling rate and limiting the measurements according to the truncation than what is obtained when measuring on the whole time series. In the present case, where the AG noise reflects quiet conditions, and does not experience aliasing, there is no preferred choice. This is rather an economic choice to be made by the operator, as a function of the actual environmental noise and taking into account the AG operational costs. The protocol should be also adapted as a function of the station and amplitude of the tidal signal, especially near the poles where the diurnal and semi-diurnal tides are missing, or near the equator where there is no diurnal tide.

\begin{tabular}{|c|c|c|c|c|c|c|c|c|}
\hline & \multicolumn{3}{|c|}{$\begin{array}{c}\text { Window : minutes around } \\
\text { extrema }\end{array}$} & \multicolumn{2}{|c|}{$1 \mathrm{drop} / 5 \mathrm{~s}$} & \multicolumn{2}{|c|}{$1 \mathrm{drop} / 10 \mathrm{~s}$} & $\begin{array}{l}\text { Available data vs. } \\
\text { whole series }\end{array}$ \\
\hline & $\begin{array}{l}\text { before } \\
\text { max. }\end{array}$ & $\begin{array}{l}\text { after } \\
\text { max. }\end{array}$ & $\begin{array}{l}\text { around } \\
\text { min. }\end{array}$ & $\begin{array}{c}\text { Error } \\
\%\end{array}$ & \# drops & $\begin{array}{c}\text { Error } \\
\%\end{array}$ & \# drops & $\%$ \\
\hline Truncated & 583.3 & 62.5 & 62.5 & 0.5 & 46675 & 0.7 & 23338 & 54 \\
\hline Whole series & & $N / A$ & & 0.3 & 85872 & 0.5 & 42936 & 100 \\
\hline
\end{tabular}

Table 1. Error on the calibration factor using the whole or truncated series as shown on Figure 3 , and of the sampling rate. The window is given as a function of the number of minutes around the maxima and minima. 


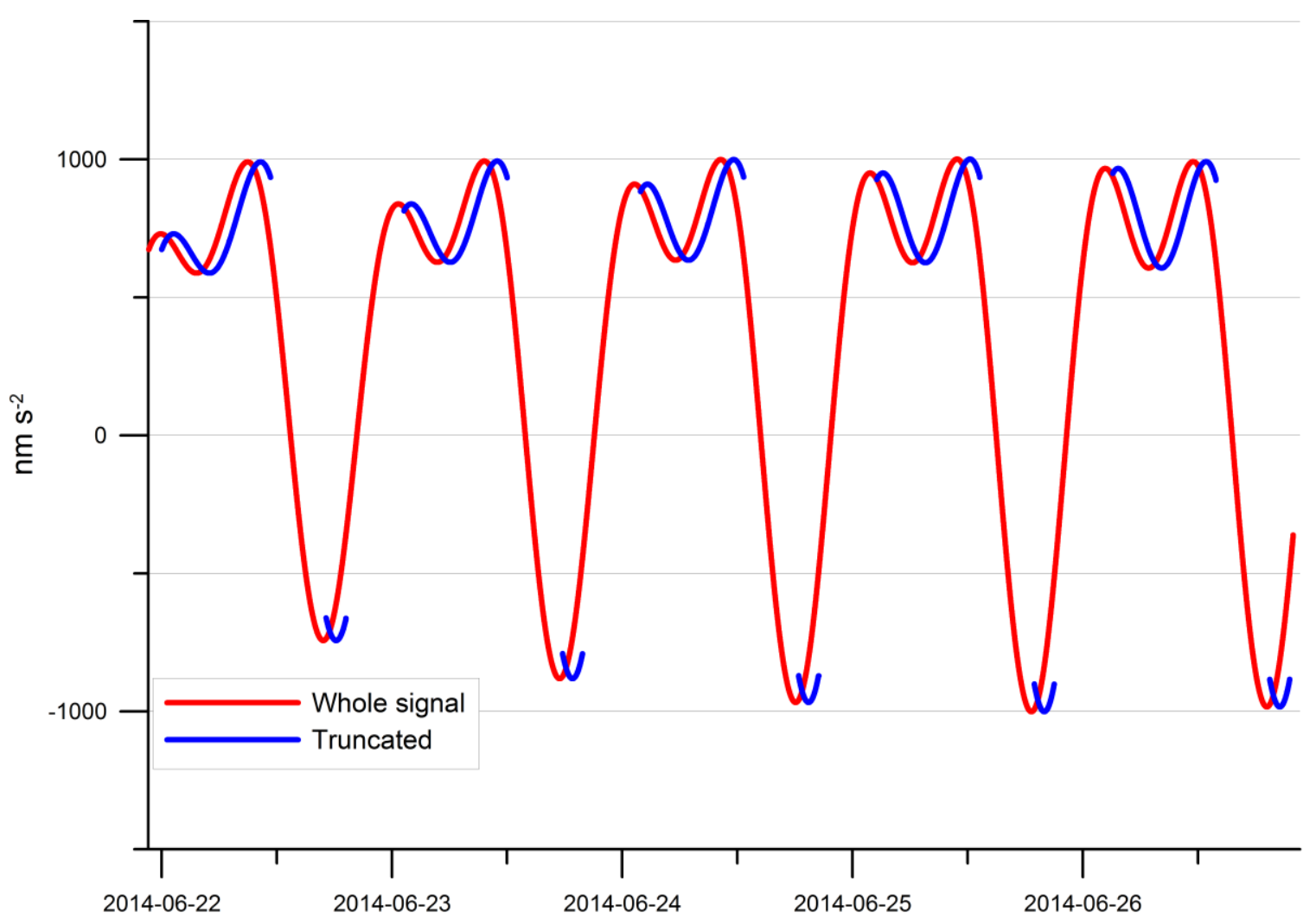

Fig. 3 Signal used to test the influence of the truncation: in red the full series, in blue the truncated series. For legibility the truncated series was shifted right

\subsection{Aliasing}

When the microseismic noise is high, causing a drop to drop scatter higher than about 150-200 $\mathrm{nm} / \mathrm{s}^{2}$, an aliasing effect influences the AG measurements. Increasing the sampling rate from 1 drop per $10 \mathrm{~s}$ to 1 drop per $5 \mathrm{~s}$ is a way to reduce this effect (Van Camp et al. 2005).

To quantify the actual effect of the aliasing on the calibration factor, a GW noise of $150 \mathrm{~nm} / \mathrm{s}^{2}$ is generated, to which a high frequency noise is added. To obtain this high frequency noise a GW noise of $1000 \mathrm{~nm} / \mathrm{s}^{2}$ amplitude is generated. Then, in the frequency domain its amplitude spectrum is multiplied by $f^{3}$, before coming back to the time domain. This allows simulating the spectral edge of the high-frequency microseismic noise. The total contribution to the rms noise amplitude in the frequency band up to $0.1 \mathrm{~Hz}$ is $231 \mathrm{~nm} / \mathrm{s}^{2}$. The PSD of the noise model has a value of $3.410^{5}$ $n m^{2} s^{-4} \mathrm{~Hz}^{-1}$ at $0.001 \mathrm{~Hz}$ and is shown in black on Figure 4. Discarding of every other sample creates a strong aliasing effect, as shown in red on Figure 4.

The ratio of the standard deviations is linked to the ratio of the PSDs according to:

$$
\frac{\sigma_{1}}{\sigma_{2}}=\sqrt{\frac{P S D_{1}}{P S D_{2}}}
$$

where $P S D_{1}$ is related to the noise level of the 1 per $10 \mathrm{~s}$ data and $P S D_{2}$ of the 1 per $5 \mathrm{~s}$ ones. Consequently, for white noise PSD levels of $1.0 \cdot 10^{7}$ and $3.4 \cdot 10^{5}\left(\mathrm{~nm} / \mathrm{s}^{2}\right)^{2} / \mathrm{Hz}$ (Figure 4), doubling the sampling rate should improve the uncertainty by a factor of $\sqrt{10^{2} / 3.4}=5.4$ provided the observation period is the same.

As the noises are colored, to quantify the actual influence, tests were made by computing the calibration factor generating 10000 noise series, with the same amplitude. As shown in Table 2, 
doubling the sampling rate increases the precision dramatically. In other words, for the same number of drops, restricting measurements to tidal extremes as on Figure 3 with a doubled rate improves the precision by a factor 5.2/1.1 $=4.7$ with respect to what would be obtained measuring continuously with a rate of 1 data every $10 \mathrm{~s}$.

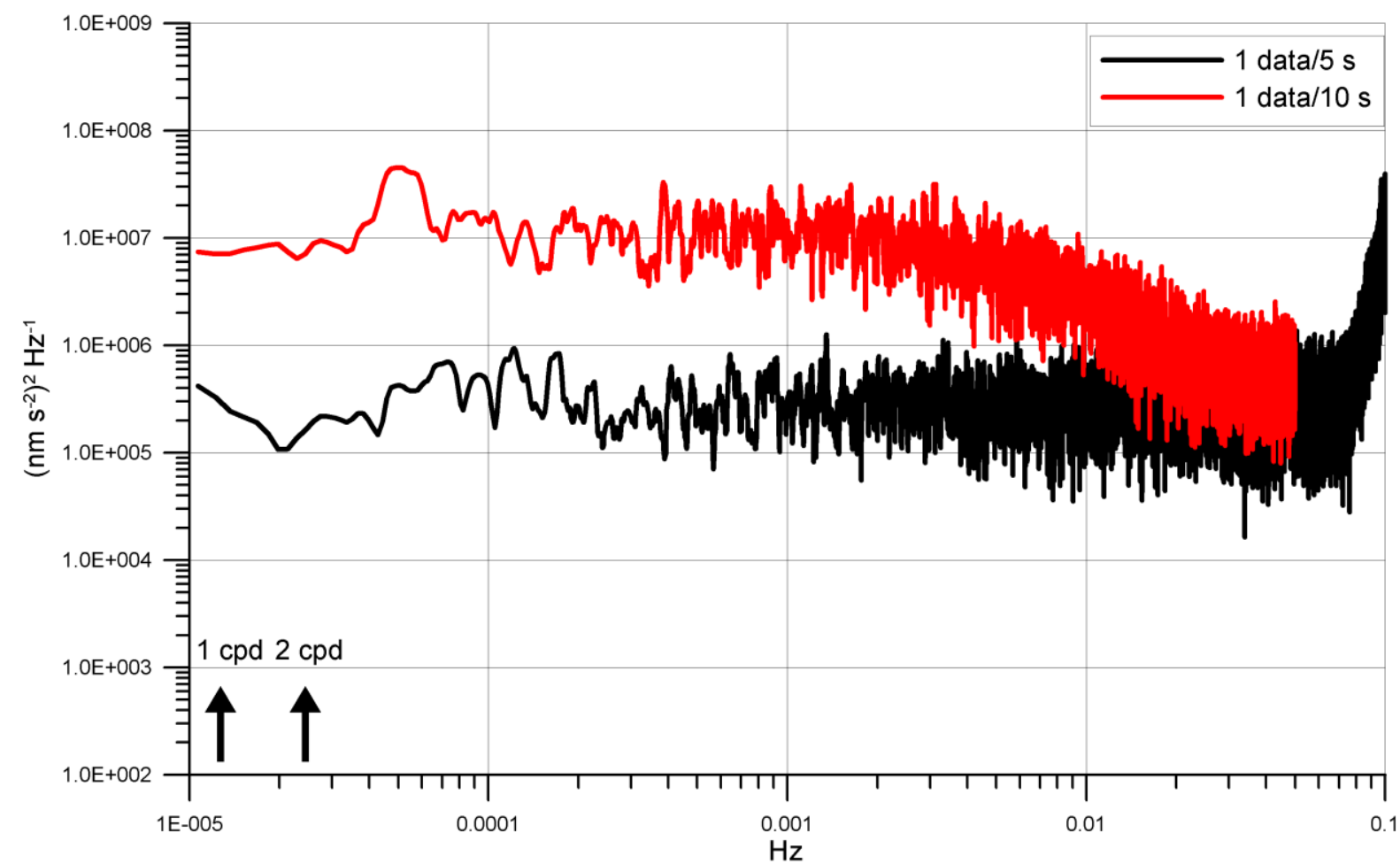

Fig. 4 PSDs of simulated AG noise. In black, using 1 data per $5 \mathrm{~s}$, in red using 1 data per $10 \mathrm{~s}$. The semi-diurnal ( 2 cycles per day) and diurnal ( 1 cycle per day) tidal frequencies are indicated by the arrows

\begin{tabular}{|l|l|l|l|l|}
\hline & \multicolumn{2}{|c|}{1 drop/5 s } & \multicolumn{2}{c|}{1 drop/10 s } \\
\hline & Error/\%o & \# data & Error/\%o & \# data \\
\hline Truncated & 1.1 & 46675 & 7.4 & 23338 \\
\hline $\begin{array}{l}\text { Whole } \\
\text { series }\end{array}$ & 0.8 & 85872 & 5.2 & 42936 \\
\hline
\end{tabular}

Table 2. Error on the calibration factor for the series shown on Figure 3, for two different sampling rates, for an $A G$ experiencing a $150 \mathrm{~nm} / \mathrm{s}^{2} \mathrm{GW}$ noise with high frequency noise of which the PSD is shown in black on Figure 4.

\section{Bias caused by the noise from superconducting gravimeter}

So far, the time series $x_{i}$ was considered as noise-free. According to previous studies (Banka and Crossley 1999; Van Camp et al. 2005; Rosat and Hinderer 2011), the SG instrumental noise is white, at the $10 \mathrm{~nm}^{2} \mathrm{~s}^{-4} \mathrm{~Hz}^{-1}$ level, corresponding to signal rms amplitude of $1 \mathrm{~nm} / \mathrm{s}^{2}$ when taking one drop every five seconds. This is lower than the AG GW noise by 80 to $120 \mathrm{~dB}$, which dominates the spectrum at frequencies larger than 1 cycle per day, as illustrated on Figure 4 . Of course, the actual signal of the SG increases at frequencies higher than $0.01 \mathrm{~Hz}$, given the structure of the geophysical noise (Peterson 1993), but most often, it remains lower than the AG white noise. 
When the microseism is high, the AG experiences aliasing as previously discussed. However, in some circumstances the PSD of the SG can reach a level close to that from the AG, at periods shorter than about $25 \mathrm{~s}$. The superspring (Niebauer et al., 1995) allows the AG to maintain a reasonable GW, while the SG experiences a dramatic increase in the high frequency noise if it is not properly lowpass filtered. This is shown on Figure 5, for a calibration experiment performed at the Membach station in May 2014. This figure shows the gravity residuals, which are the gravity signals after correcting for tidal and atmospheric effects. The AG drop to drop scatter amounts to an rms amplitude of $62 \mathrm{~nm} / \mathrm{s}^{2}$, equivalent to $77000 \mathrm{~nm}^{2} \mathrm{~s}^{-4} \mathrm{~Hz}^{-1}$ when taking one drop every ten seconds, which can be considered as low noise condition, on the one hand. On the other hand, the SG experienced several perturbations due to moderate and strong earthquakes around the Pacific Ocean, with magnitudes $\mathrm{Mw}$ ranging 5.5-6.3. Note that those events are still too small to be observable in the AG series.

In that case, unless the SG signal is correctly low-passed, the classical LSQ theory cannot be applied anymore, as $x$ is not error-free. Let us consider that the SG time series $x_{i}$ includes an independent measurement error $\eta_{i}$ :

$$
x_{i}=\tilde{x}_{i}+\eta_{i}
$$

where $\tilde{x}_{i}$ would be the SG output voltage in the error free case.

We then have

$$
y_{i}=\beta\left(x_{i}-\eta_{i}\right)+\varepsilon_{i}=\beta x_{i}+u_{i}
$$

with

$$
u_{i}=\varepsilon_{i}-\beta \eta_{i}
$$

As $x$ and $\eta$ are not independent, considering that $\tilde{x}, \varepsilon, \eta$ are independent, we have:

$$
\operatorname{Cov}(x, u)=\operatorname{Cov}(\tilde{x}+\eta, \varepsilon-\beta \eta)=-\beta \operatorname{var}(\eta)
$$

Consequently, the estimator of the calibration factor becomes:

$$
\hat{\beta}=\frac{\operatorname{cov}(x, y)}{\operatorname{var}(x)}=\beta\left(1-\frac{\operatorname{var}(\eta)}{\operatorname{var}(x)}\right)=\beta \cdot \frac{\operatorname{var}(\tilde{x})}{\operatorname{var}(\tilde{x})+\operatorname{var}(\eta)}
$$

We see that $\hat{\beta}$ is systematically underestimated, by a factor $\frac{\operatorname{var}(\tilde{x})}{\operatorname{var}(\tilde{x})+\operatorname{var}(\eta)}$. Note that this effect does not add variance to the estimator, but a systematic negative bias of the estimation. This phenomenon is known as attenuation or regression dilution bias (Hutcheon et al. 2010).

In many cases evaluating this bias is not straightforward (Frost and Thompson 2000). Fortunately, the attenuation bias is easy to determine in case of gravity measurements, given that $\tilde{x}$ is essentially the tidal signal, and $\eta$ the SG residual, obtained after removing a synthetic tide and correcting the atmosphere effect. Application of an appropriate low-pass filter to the SG series will mitigate this bias.

Another SG instrumental effect is the time lag. However, our tests show that an uncorrected lag as large as $30 \mathrm{~s}$ influences the calibration factor at a level smaller than 0.1 per mille (see also Meurers 2002). 


\section{Simulation}

We consider the first ten days of the Membach time series shown on Figure 1, of which the rms amplitude equals $542.8 \mathrm{~nm} / \mathrm{s}^{2}$. To mimic the SG noise, we added a red noise, a violet noise and an ultraviolet noise. To obtain a red noise we generate a GW noise of $2 \mathrm{~nm} / \mathrm{s}^{2}$ amplitude. Then, in the frequency domain its amplitude spectrum is multiplied by $f^{-1}$, before coming back to the time domain. Similarly, the violet and ultraviolet noises are obtained from 20 and $30 \mathrm{~nm} / \mathrm{s}^{2} \mathrm{GW}$ noises, of which the amplitude spectra are multiplied by respectively $f$ and $f^{2}$. The total contribution to the rms noise amplitude in the whole frequency band up to $0.05 \mathrm{~Hz}$ is $7.9 \mathrm{~nm} / \mathrm{s}^{2}$. The PSD of the noise model has a value of $2.4 \mathrm{~nm}^{2} \mathrm{~s}^{-4} \mathrm{~Hz}^{-1}$ at $0.001 \mathrm{~Hz}$ and is shown in red on Figure $5 \mathrm{~b}$, together with the PSD of the SG residual of Membach. The AG noise is modeled by a $77000 \mathrm{~nm}^{2} \mathrm{~s}^{-4} \mathrm{~Hz}^{-1} \mathrm{GW}$ noise (equivalent to $62 \mathrm{~nm} / \mathrm{s}^{2}$ standard deviation at a period of $10 \mathrm{~s}$ ).

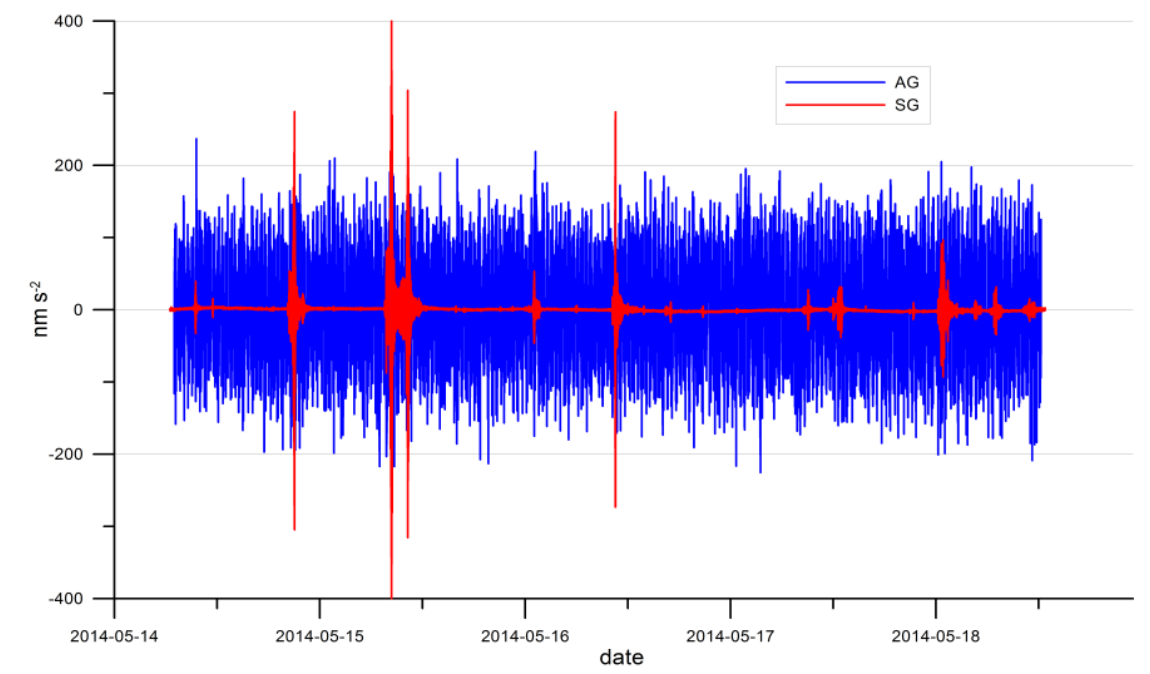

(a)

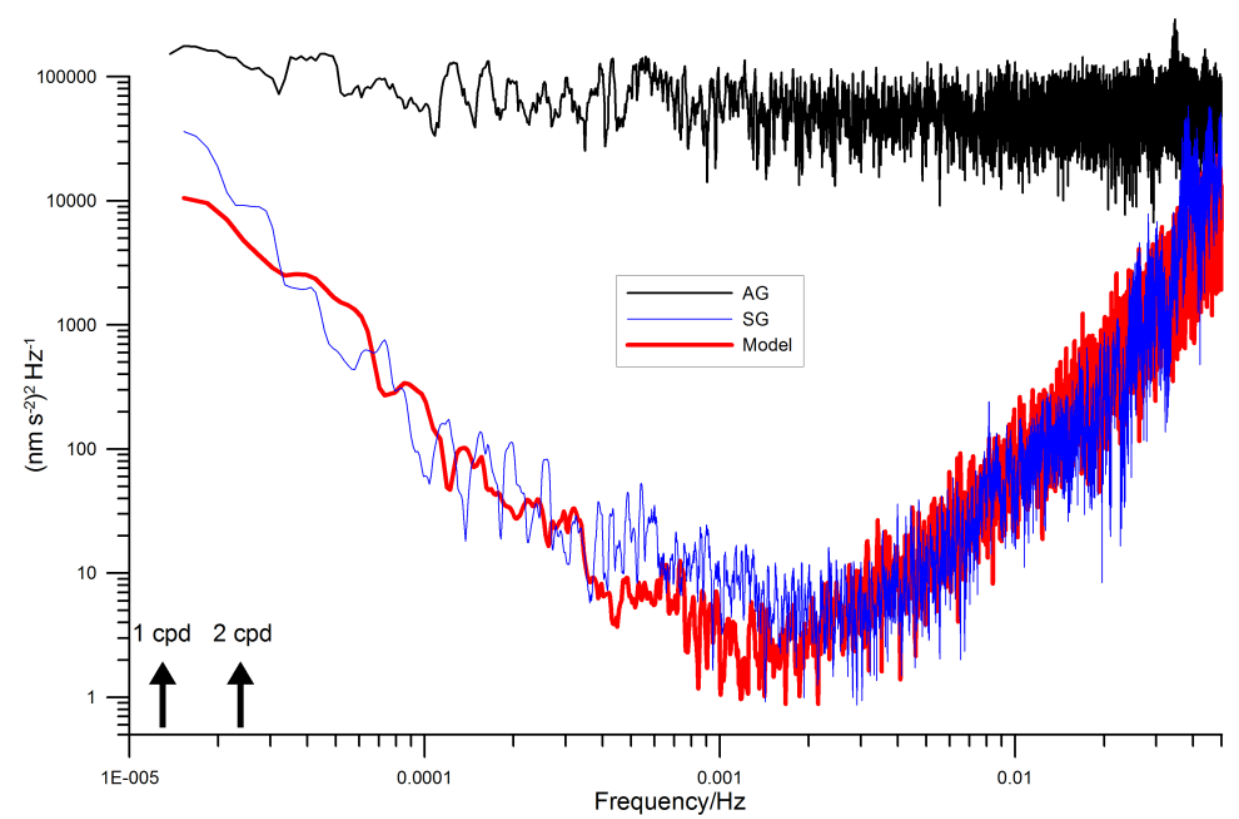

(b)

Fig. 5(a) SG and AG residuals and Fig. 5(b) their PSDs at Membach (SG data taken when the AG drops are available) from 2014-0514 01:00 to 201405-18 14:46, 1 drop/10 s, 100 drops/set, 2 sets/hour; the PSD of the noise model used in our simulations is also shown in red. The semi-diurnal ( 2 cycles per day) and diurnal ( 1 cycle per day) tidal frequencies are indicated by the arrows 
The calibration factor was calculated using the LSQ approach; this was repeated ten thousand times, generating a different random noise for each run.

The observed distribution from the simulation has a $0.31 \mathrm{~nm} / \mathrm{s}^{2} / \mathrm{V}$ precision, with a $783.82 \mathrm{~nm} / \mathrm{s}^{2} / \mathrm{V}$ mean. This is lower than the expected factor of $784 \mathrm{~nm} / \mathrm{s}^{2} / \mathrm{V}$ by $0.22 \%$, consistent with the bias predicted by eq. (7):

$$
\frac{542.8^{2}}{542.8^{2}+7.9^{2}}=0.99978
$$

This result is more than 4 times smaller than the targeted per mille level, but this is a systematic bias which is not accounted for in the error bars provided by the LSQ process.

To ensure that the bias remains at a negligible level compare to the target per mille level, it makes sense to achieve a calibration for which the bias is smaller than $0.1 \%$.

According to eq. (7), we need

$$
\frac{\sigma_{\eta}}{\sigma_{\tilde{x}}}<\sqrt{\frac{1-0.9999}{0.9999}}=10^{-2}
$$

where $\eta$ is the SG residual, estimated after removing a synthetic tide and correcting the atmosphere effect using a linear admittance of $-3.3 \mathrm{nms}^{-2} / \mathrm{hPa}$, and $\tilde{x}$ is the actual tidal signal.

The formula (8) holds for white noise, our test shows that this is the high frequency noise of the SG which might be high enough to bias the estimate of the calibration factor. Table 3 provides the attenuation factor for a 100 day time series at Membach as a function of the noise level.

\begin{tabular}{|l|l|}
\hline Noise stdv $\left[\mathrm{nms}^{-2}\right]$ & Attenuation factor (eq. (7)) \\
\hline 5 & 0.9999 \\
\hline 50 & 0.9935 \\
\hline 100 & 0.9744 \\
\hline 200 & 0.9048 \\
\hline 500 & 0.6032 \\
\hline
\end{tabular}

Table 3. Attenuation factor estimated according to eq. (7) for rms amplitude of $616 \mathrm{~nm} / \mathrm{s}^{2}$ for the tidal signal at Membach, as a function of the rms amplitude of the noise $\eta$ affecting the $\tilde{x}_{i}$ values.

\section{Conclusions}

We demonstrated that the per mille precision can be reached within 48 hours by measuring at spring tides and by increasing the AG sampling rate. This is shorter than what is reported in previous empirical studies (Hinderer et al., 1998; Francis et al., 1998; Rosat et al., 2009), but supports the result of Francis (1997). We also showed that the error decrease with $1 / \sqrt{N}$ is not correct, given the tidal modulation effect. We then evidenced that, if the standard deviation of the noise affecting the SG is at least 100 times lower than the rms amplitude of the tidal signal used to compute the calibration factor, then the attenuation bias remains lower than the $0.1 \%$ level. To mitigate this bias, a least square LSQ filter with cutoff frequency of $0.05 \mathrm{~Hz}$ and length of $60 \mathrm{~s}$ is an appropriate choice, given that the macroseismic noise is strong above $0.05 \mathrm{~Hz}$. The cutoff frequency must remain high enough not to remove a common signal to AG and SG.

Filtering would not help in other cases, for example if the noise is induced by spikes or steps, as they contaminate the whole frequency band. Editing carefully the AG and SG time series to remove 
earthquakes, spikes and other disturbance is the minimum to be done before applying the LSQ process (Hinderer et al. 2007). Note that at frequencies smaller than about one cycle per day, the geophysical red noise affects the SG and AG in the same way (Van Camp et al. 2005) and does not bias the determination of the calibration factor.

The calibration precision of SGs operating in the framework of the Global Geodynamics Project varies about a few per mille and different calibration experiments for the same SG can differ by more than 1\%o (Rosat et al. 2009; Meurers et al. 2012; Virtanen et al. 2014). This is expected given that the per mille value represents the one sigma level, such that $32 \%$ of the calibration factors lie outside the error bars, assuming that the attenuation bias only plays a negligible role. Assuming that the SG calibration factor remains stable, to ensure a robust calibration factor, with error well below the $1 \%$ level, we need averaging over several experiments, as shown by Rosat et al. (2009) and Virtanen et al. (2014). Considering that a calibration experiment is performed at the one per mille level, using more than 30,000 drops, we can assume an infinite number of degrees of freedom for the Student's t-distribution. If we take a risk that 5 times in 100 the error in the calibration estimate will be at more than the 1 per mille level, 4 experiments will be required, or 16 at the 0.5 per mille level, or 43 at the 0.3 per mille level (Natrella 1963). For a risk of 1 time in 100, the number of experiments becomes 7, 27 and 74, respectively.

This is not contradicted by Figure 6, which represents the calibration factors determined by performing 13 experiments on the Austrian SG GWR-C025, as well as the evolution of the average factor as a function of the experiments. These experiments were done using JILAg (Faller et al. 1983) and FG5 AGs. The first three experiments lasted over more than 3.5 days, thereafter over 4-8 days. As shown by the light red curve, 3 factors differ by one per mille or more (050620; 111121 and 120609); however, the calibration factor stabilizes well below the $1 \%$ o level after the second experiment (dark blue line).

In the future, atom absolute gravimeters may change that picture as they do not rely on a mechanical process, and can thus be operated continuously (de Angelis et al. 2009). In that case for the same conditions as illustrated on Figure 1, it would require 21 days to achieve the $0.33 \%$ o level, which would ensure a calibration factor at the $1 \%$ level with a $99.7 \%$ confidence interval. If the GW noise of future absolute gravimeters decreases, this precision could be obtained in less than 21 days.

When calibration factors are discussed, the amplitude of the tidal signal, the duration of the AG measurements, the attenuation bias as well as the AG sampling rate and the number of drops should be provided.

Finally, even if a calibration experiment could be performed for months, other factors may limit the precision at a level better than a few tenths of per mille: small instabilities in the SG calibration factor, changing drifts, or possible non-linearity in the sensors. Other factors such as the calibration of the AG atom clock, tilts of the instrument or changes in the refraction index or magnetic field (see Niebauer et al. (1995) for a comprehensive review of the possible sources of error), all are at a level smaller than $10^{-8}$ and are not presently of concern. 


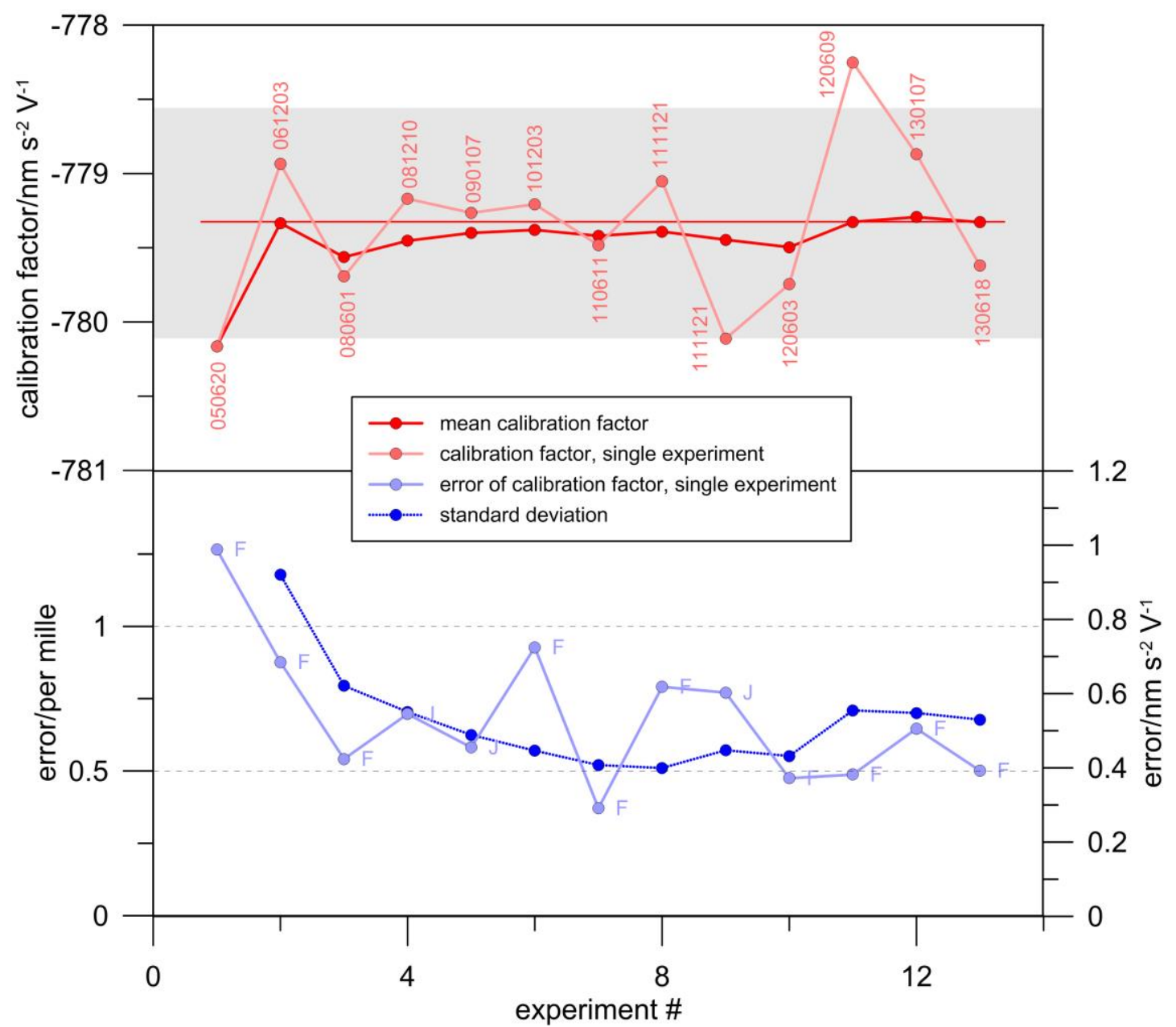

Fig. 6 Calibration factors of the SG GWR-C025 during different experiments using an FG5 (F) and a JILAg (J) AG. Light red: single results, light blue: their errors associated to the LSQ process. Gray: the $1 \%$ range around the average. With the exception of 3 experiments, all results deviate from the overall weighted average by less than $1 \%$. All but the first formal error (light blue) are well below $1 \%$, which is equivalent to $0.79 \mathrm{~nm} \mathrm{~s}^{-2} \mathrm{~V}^{-1}$ for this SG. The dark red shows how the weighted average develops with increasing number of experiments. The dotted dark blue line shows how the standard deviation from the average develops

\section{Acknowledgements}

The work of OdV is financially supported by the Institut Universitaire de France. We thank the editor Roland Klees and three anonymous reviewers for their fruitful comments.

\section{Bibliography}

Achilli V, Baldi P, Casula G, Errani M, Focardi S, Guerzoni M, Palmonari F, Raguni G (1995) A calibration system for superconducting gravimeters. Bull Géod 69:73-80

Baker TF, Bos MS (2003) Validating Earth and ocean tide models using tidal gravity measurements. Geophys J Int. doi: 10.1046/j.1365-246X.2003.01863.x

Banka D, Crossley D (1999) Noise levels of superconducting gravimeters at seismic frequencies. Geophys J Int. doi: 10.1046/j.1365-246X.1999.00913.x 
Crossley D, Hinderer J (2009) A review of the GGP network and scientific challenges. J Geodyn 48:299-304

de Angelis M, Bertoldi A, Cacciapuoti L, Giorgini A, Lamporesi G, Prevedelli M, Saccorotti G, Sorrentino F, Tino GM (2009) Precision gravimetry with atomic sensors. Meas Sci Technol. doi:10.1088/0957-0233/20/2/022001

Faller JE, Guo YG, Geschwind J, Niebauer TM, Rinker RL, Xue J (1983) The JILA portable absolute gravity apparatus. Bull Inf Bur Grav Int 53:87-97

Francis O (1997) Calibration of the C021 superconducting gravimeter in Membach (Belgium) using 47 days of absolute gravity measurements. Int Ass Geod Symp 117:212-218

Francis O, Niebauer TM, Sasagawa G, Klopping F, Gschwind J (1998) Calibration of a superconducting gravimeter by comparison with an absolute gravimeter FG5 in Boulder. Geophys Res Lett 25:10751078

Francis O, Hendrickx M (2001) Calibration of the LaCoste-Romberg 906 by Comparison with the Superconducting Gravimeter C021 in Membach (Belgium). J Geod Soc Japan 47(1):16-21.

Frost C, Thompson S (2000) Correcting for regression dilution bias: comparison of methods for a single predictor variable. J R Statist Soc A 163(2):173-189

Hinderer J, Florsch N, Mäkinen J, Legros H, Faller JE (1991) On the calibration of a superconducting gravimeter using absolute gravity measurements. Geophys J Int doi:10.1111/j.1365246X.1991.tb03907.x

Hinderer J, Amalvict M, Florsch N, Francis O, Makinen J (1998) On the calibration of superconducting gravimeters with the help of absolute gravity measurements. In Ducarme B, Pâquet P (eds) Proc XIII Int Symp on Earth Tides, Bruxelles, pp 557-564

Hinderer J, Crossley D, Warburton RJ (2007), Gravimetric Methods - Superconducting Gravity Meters. In: Herring T, Schubert G (eds) Treatise on Geophysics, 3, pp 65-122

Hutcheon JA, Chiolero A, Hanley JA (2010) Random measurement error and regression dilution bias. BMJ. doi: 10.1136/bmj.c2289

Imanishi Y, Higashi T, Fukuda Y (2002) Calibration of the superconducting gravimeter T011 by parallel observation with the absolute gravimeter FG5 \#210-a Bayesian approach. Geophys J Int doi: 10.1046/j.1365-246X.2002.01806.x

Meurers B (2001) Superconducting gravimetry in geophysical research today. J Geodet Soc of Japan 47:300-307

Meurers B (2002) Aspects of gravimeter calibration by time domain comparison of gravity records. Bull. Inf. Marées Terrestres 135:10643-10650

Meurers B (2012) Superconducting Gravimeter Calibration by Collocated Gravity Observations: Results from GWR C025. Int J Geophys doi:10.1155/2012/954271

Natrella MG (1963) Experimental Statistics. National Bureau of Standards Handbook 91, Aug; 1, 1963, US Dep Of Commerce 
Niebauer TM, Sasagawa G, Faller J, Hilt R, Klopping F (1995) A new generation of absolute gravimeters. Metrologia. doi:10.1088/0026-1394/32/3/004

Peterson J (1993) Observations and modeling of seismic background noise. US Geol Surv Rep. 93322 , Albuquerque, NM

Richter B, Wilmes H, Nowak I (1995) The Frankfurt calibration system for relative gravimeters. Metrologia 32:217-223

Rosat S, Boy JP, Ferhat G et al. (2009) Analysis of a 10-year (1997-2007) record of time-varying gravity in Strasbourg using absolute and superconducting gravimeters: new results on the calibration and comparison with GPS height changes and hydrology. J Geodyn 48(3-5):360-365

Rosat S, Hinderer J (2011) Noise Levels of Superconducting Gravimeters: Updated Comparison and Time Stability. Bull Seism Soc Am. doi: 10.1785/0120100217

Van Camp M, Wenzel HG, Schott P, Vauterin P, Francis O (2000) Accurate transfer function determination for superconducting gravimeters. Geophys Res Lett 27:37-40

Van Camp M, Williams SDP, Francis O (2005) Uncertainty of absolute gravity measurements. J Geophys Res. doi:10.1029/2004JB003497

Virtanen H, Bilker-Koivula M, Mäkinen J, Näränen J, Raja-Halli A, Ruotsalainen H (2014) Comparison between measurements with the superconducting gravimeter T020 and the absolute gravimeter FG5-221 at Metsähovi, Finland in 2003-2012. Bull Inf Marées Terrestres 148:11923-11928 